\title{
Gene Interactions and Structural Brain Change in Early-Onset Alzheimer's Disease Subjects Using the Pipeline Environment
}

\author{
Seok Woo Moon ${ }^{\bowtie}$, Ivo D. Dinovi,3, Alen Zamanyan², Ran Shi², Alex Genco², Sam Hobel², \\ Paul M. Thompson ${ }^{2}$, Arthur W. Toga ${ }^{2}$, and the Alzheimer's Disease Neuroimaging Initiative (ADNI) \\ 'Department of Psychiatry, Konkuk University School of Medicine, Chungju, Republic of Korea \\ ${ }^{2}$ Laboratory of Neuro Imaging, Institute for Neuroimaging and Informatics, University of Southern California, Los Angeles, CA, USA \\ ${ }^{3}$ Statistics Online Computational Resource, UMSM, University of Michigan, Ann Arbor, MI, USA
}

\begin{abstract}
Objective This article investigates subjects aged 55 to 65 from the Alzheimer's Disease Neuroimaging Initiative (ADNI) database to broaden our understanding of early-onset (EO) cognitive impairment using neuroimaging and genetics biomarkers.

Methods Nine of the subjects had EO-AD (Alzheimer's disease) and 27 had EO-MCI (mild cognitive impairment). The 15 most important neuroimaging markers were extracted with the Global Shape Analysis (GSA) Pipeline workflow. The 20 most significant single nucleotide polymorphisms (SNPs) were chosen and were associated with specific neuroimaging biomarkers.

Results We identified associations between the neuroimaging phenotypes and genotypes for a total of 36 subjects. Our results for all the subjects taken together showed the most significant associations between rs7718456 and L_hippocampus (volume), and between rs7718456 and R_hippocampus (volume). For the 27 MCI subjects, we found the most significant associations between rs6446443 and R_superior_frontal_gyrus (volume), and between rs17029131 and L_Precuneus (volume). For the nine AD subjects, we found the most significant associations between rs16964473 and L_rectus gyrus (surface area), and between rs12972537 and L_rectus_gyrus (surface area).

Conclusion We observed significant correlations between the SNPs and the neuroimaging phenotypes in the 36 EO subjects in terms of neuroimaging genetics. However, larger sample sizes are needed to ensure that the effects will be detectable for a reasonable false-positive error rate using the GSA and Plink Pipeline workflows.

Psychiatry Investig 2015;12(1):125-135
\end{abstract}

Key Words Alzheimer's disease, Early-onset, ADNI, Mild cognitive impairment, Memory, Neuroimaging, Genetics.

\section{INTRODUCTION}

Alzheimer's disease (AD) is the most common form of dementia and has a strong genetic component, with up to $80 \%$ heritability, as estimated from twin-concordance studies. ${ }^{1,2} \mathrm{It}$ is generally divided into two main subtypes. One subtype is the autosomal-dominant, familial early-onset $\mathrm{AD}$ (EO-AD). A very small percentage of people who develop $\mathrm{AD}$ have the EO variety, which is classified as the beginning of the disease presentation before the age of 65 . Researchers have found out

Received: November 5, 2013 Revised: February 2, 2014

Accepted: February 3, 2014 Available online: January 12, 2015

$\triangle$ Correspondence: Seok Woo Moon, MD, PhD

Dementia Center, Department of Neuropsychiatry, Konkuk University School of Medicine, 82 Gugwon-daero, Chungju 380-704, Republic of Korea Tel: +82-43-840-8990, Fax: +82-43-857-1380, E-mail: hessem@naver.com

(c) This is an Open Access article distributed under the terms of the Creative Commons Attribution Non-Commercial License (http://creativecommons.org/licenses/bync/3.0) which permits unrestricted non-commercial use, distribution, and reproduction in any medium, provided the original work is properly cited. three genes whose mutations cause EO-AD. These are $\mathrm{APP}^{3}$ (amyloid precursor protein) on chromosome 21; $\mathrm{PS}^{4}$ (presenilin 1) on chromosome 14; and PS2 $2^{5,6}$ (presenilin 2) on chromosome 1 . The other group is sporadic, non-familial AD, most commonly termed "late-onset AD" (LO-AD), which is defined with the disease presentation after the age of 65 .

Genetic studies have provided significant insights into the molecular basis of AD. Rare hereditary EO forms of the disease have been linked to mutations of three different genes, as mentioned. These mutations explain, however, less than $1 \%$ of all cases of AD. ${ }^{7}$ Some people who have EO-AD do not have mutations in any of these three genes. That suggests that $\mathrm{EO}-\mathrm{AD}$ is linked to other genetic mutations that have not been identified yet.

In this paper, "neuroimaging genetics" refers to the use of computationally-derived neuroanatomical, functional, or connectivity imaging markers as phenotype assays to evaluate the genetic variation. Recent and ongoing advances in neuro- 
imaging genetics have made it possible to scan populations with multimodality neuroimaging to collect genome-wide data $^{8,9}$ and to study the influence of genetic variation on the brain structure and function. ${ }^{10-12}$ Quantitative trait (QT) association studies have been shown to have increased the statistical power and thus, decreased the sample size requirements. ${ }^{13}$ In addition, neuroimaging phenotypes may be closer to the underlying biological etiology of the disease, which makes it easier to identify underlying genes. ${ }^{10}$ This study focuses on analyzing gene interactions and collective genome effects on the brain structure in ADNI EO-AD and EO-mild cognitive impairment (MCI) data. Specifically, this study uses existing LONI computational tools and techniques (e.g., the LONI Probabilistic Brain Atlas, ${ }_{1}^{14}$ BrainParser, ${ }^{15}$ and the LONI Pipeline environment ${ }^{16,17}$ ) to study interrelations between phenotypes, genotypes, and biomedical neuroimaging features in the subjects from ADNI.

A broad search for genes, SNPs, and alleles was used holistically to identify their associations with brain shape changes and subject phenotypes. We investigated whether or not there are collective multi-gene patterns that are significantly associated with imaging biomarkers. SNPs and other types of polymorphisms in single genes such as APOE have been related to neuroimaging measures in both healthy controls and cognitive impairment (such as $\mathrm{MCI}$ and $\mathrm{AD}$ ) patients; however, a single gene or a few imaging measures may be insufficient to understand the multiple mechanisms and imaging manifestations of these complex diseases. Thus, we used a genomewide association study (GWAS) to relate high-throughput SNP data to large-scale neuroimaging data. We conducted a GWAS on the EO-AD and EO-MCI subjects to study the genes that influence the brain structure across all the brain regions.

This article uses data from subjects aged 55 to 65 to broaden our understanding of $\mathrm{EO}$ cognitive impairment (especially $\mathrm{EO}-\mathrm{AD}$ and $\mathrm{EO}-\mathrm{MCI}$ ) in terms of neuroimaging genetics. We hypothesize that some specific SNPs that discriminate between EO-AD and EO-MCI subjects may be extracted in terms of GWAS, and EO cognitive impairment patients have different ROIs specific to EO cognitive impairment. Thus, we can associate the two results and identify a significant relationship between them. Several studies have been conducted on the relationship between neuroimaging phenotypes and genetic variants, but nothing has been reported yet on either shape-based neuroimaging measures or EO cognitive impairment subjects. ${ }^{10,18,19}$ Therefore, we are attempting to expand the narrow scope of studies, in terms of EO cognitive impairment, in the field of neuroimaging genetics.

\section{METHODS}

\section{ADNI data}

ADNI was launched in 2003 by the National Institute on Aging (NIA), the National Institute of Biomedical Imaging and Bioengineering (NIBIB), the Food and Drug Administration (FDA), private pharmaceutical companies, and nonprofit organizations as a $\$ 60$ million, five-year public-private partnership. The primary goal of ADNI has been to test if serial magnetic resonance imaging (MRI) and positron emission tomography (PET) results, other biological markers, and clinical and neuropsychological assessment results can be combined to measure the progression of $\mathrm{MCI}$ and early $\mathrm{AD}$. The determination of sensitive and specific markers of very early $\mathrm{AD}$ progression is intended to aid researchers and clinicians to develop new treatments and to monitor their effectiveness, as well as to lessen the time and cost of clinical trials. The Principle Investigator of this initiative is Michael W. Weiner, M.D., of VA Medical Center and the University of California San Francisco. ADNI is the result of the efforts of many co-investigators from a broad range of academic institutions and private corporations, and its subjects were recruited from over 50 sites across the U.S. and Canada. The initial goal of ADNI was to recruit 800 adults aged 55 to 90 to participate in the research, in which approximately 200 cognitively normal older individuals were to be followed for three years, 400 people with MCI were to be followed for three years, and 200 people with early AD were to be followed for two years. For up-to-date information, see http://www.adniinfo.org.

\section{Study participants}

Data (ADNI study design): The participants were screened, enrolled, and followed up prospectively according to the ADNI study protocol described in detail elsewhere..$^{20}$ The degree of clinical severity of the condition of each participant was evaluated with an annual semi-structured interview. This interview generated an overall Clinical Dementia Rating (CDR) score and the CDR Sum of Boxes. ${ }^{21}$ The Mini-Mental State Examination (MMSE) for each structural MRI scan was also conducted. APOE genotyping was determined using DNA obtained from the subjects' blood samples and was performed at the University of Pennsylvania.

The participants were selected from the ADNI database if they were classified at the baseline as 1) cognitively normal control individuals with a CDR score of $0 ; 2$ ) patients with MCI with an MMSE score of between 24 and 29, a subjective memory complaint verified by an informant, objective memory loss as measured by the Wechsler Memory Scale-Revised, a CDR score of 0.5 , absence of significant levels of impair- 
ment in other cognitive domains, essentially preserved activities of daily living, and absence of dementia at the time of the baseline MRI scan, and who were classified as having the amnestic type of MCI based on the revised MCI criteria; and 3) patients with $\mathrm{AD}$ who met the criterion for probable $\mathrm{AD}$ (a CDR score of 1 ).

These $36 \mathrm{EO}-\mathrm{AD}$ and EO-MCI subjects were chosen from among all the subjects in the ADNI database (DB) who were aged between 55 and 65 as of September 2010. Nine of them (four male and five female) had EO-AD, and 27 (15 male and 12 female) had EO-MCI.

\section{Genotyping and PLINK (genotype data)}

Individual-level genotype data in the ADNI DB were downloaded and merged to form a single dataset that contained genome-wide information for 36 individuals. Genetic analyses were performed using PLINK version 1.09 (http:// pngu.mgh.harvard.edu/ purcell/plink/).

Blood samples were obtained from each participant and sent to Pfizer for DNA extraction. All the DNA extractions and genotyping were blinded to the group assignments. TGen genotyped the blinded DNA using the Illumina Human610Quad BeadChip. Approximately 200 ng of DNA was used to genotype each subject sample according to the manufacturer's protocol (Illumina, San Diego, CA, USA). After amplification, fragmentation, and hybridization, the specifically hybridized DNA was fluorescently labeled with a single base extension reaction and detected using a BeadArray scanner. Non-specifically hybridized fragments were removed by washing them off, and the remaining specifically hybridized DNA were processed for the single base extension reaction, stained, and imaged on an Illumina Bead Array Reader. The normalized bead intensity data obtained for each sample were loaded into the Illumina BeadStudio 3.2 software, which generated SNP genotypes from fluorescent intensities using the manufacturer's default cluster settings. The data analyzed with BeadStudio are publicly available on the LONI website (http:// ADNI.loni.usc.edu). The raw genotypic data were imported into a genome-wide data management system, i.e., SNPLims, to allow the export of user-defined formats compatible with the genetic programs used for the statistical analysis.

The quality control (QC) procedures were implemented for the genome-wide data. We performed all data analyses and QC procedures using the PLINK software package (http:// pngu.mgh.harvard.edu/purcell/plink/), release v1.09. SNPs were excluded from the imaging genetics analysis if they could not meet any of the following criteria: 1) a call rate per SNP $>90 \%$; 2) a minor allele frequency (MAF) $>10 \%$; and 3) a Hardy-Weinberg equilibrium test result of $\mathrm{p}>0.01$. A total of 367,899 SNPs were finally included in the analyses. A total of
36 EO individuals enrolled had genotype data available for analysis without exclusion.

\section{MRI data (ADNI data)}

We used the data obtained from the ADNI DB (http:// www.loni.usc.edu/ADNI). The ADNI MRIs analyzed here were the baseline, screening, or follow-up scans at the entry to the study. They were acquired at multiple sites using the GE Health Care (Buckinghamshire, England), Siemens Medical Solutions USA (Atlanta, Georgia), or Philips Electronics 1.5 T (Philips Electronics North America; Sunnyvale, California) system. $^{22}$ Two high-resolution T1-weighted volumetric-magnetization-prepared $180^{\circ}$ radiofrequency pulses and rapid gradient-echo scans were collected from each study participant and normalized for intensity inhomogeneities, nonbrain tissue was removed, and subcortical white matter and deep gray matter volumetric structures were segmented. ${ }^{23,24}$ The raw Digital Imaging and Communications in Medicine images were downloaded from the public ADNI site (http:// www.loni.usc.edu/ADNI/Data/index.shtml). All the MRIs were processed according to previously published methods $\mathrm{s}^{25}$ using the FreeSurfer version 4.1.0 software package (http:// surfer.nmr.mgh.harvard.edu). The intensity gradients were followed outward from the white matter surface to find the gray matter surface (gray-cerebrospinal fluid boundary). ${ }^{26,27}$

\section{LONI pipeline environment}

LONI Pipeline ${ }^{16,28}$ is a graphical workflow environment that allows the design, execution, validation, and provenance of complex heterogeneous computational protocols. In this paper, the Pipeline environment was used to investigate the interrelations between the subjects' phenotypes, genotypes, and biomedical imaging markers, including volumetric and shapebased measures of their brain structure. For the EO ADNI subjects, our analysis protocols included automatic imaging feature extraction, geometric modeling, and statistical analysis of various global and regional anatomical measures.

The Global Shape Analysis ${ }^{1,2}$ Pipeline workflow ${ }^{16}$ provides an automated protocol for high-throughput data preprocessing (e.g., skull-stripping ${ }^{29}$ and volumetric registration ${ }^{30}$ ), brain anatomical parcellation into 56 ROIs, ${ }^{14,15}$ and extraction of shape and volume measures (average mean curvature, surface area, volume, shape index, and curvedness) between the group statistical analyses of the shape regional differences, as well as generation of 3D scene files that illustrate the statistically significant regional anatomical differences between the EO-AD and EO-MCI cohorts.

Figure 1 illustrates the completed GSA workflow for this study and one $3 \mathrm{D}$ scene file that corresponded to the ROI volume metric. We used this GSA Pipeline workflow to ob- 


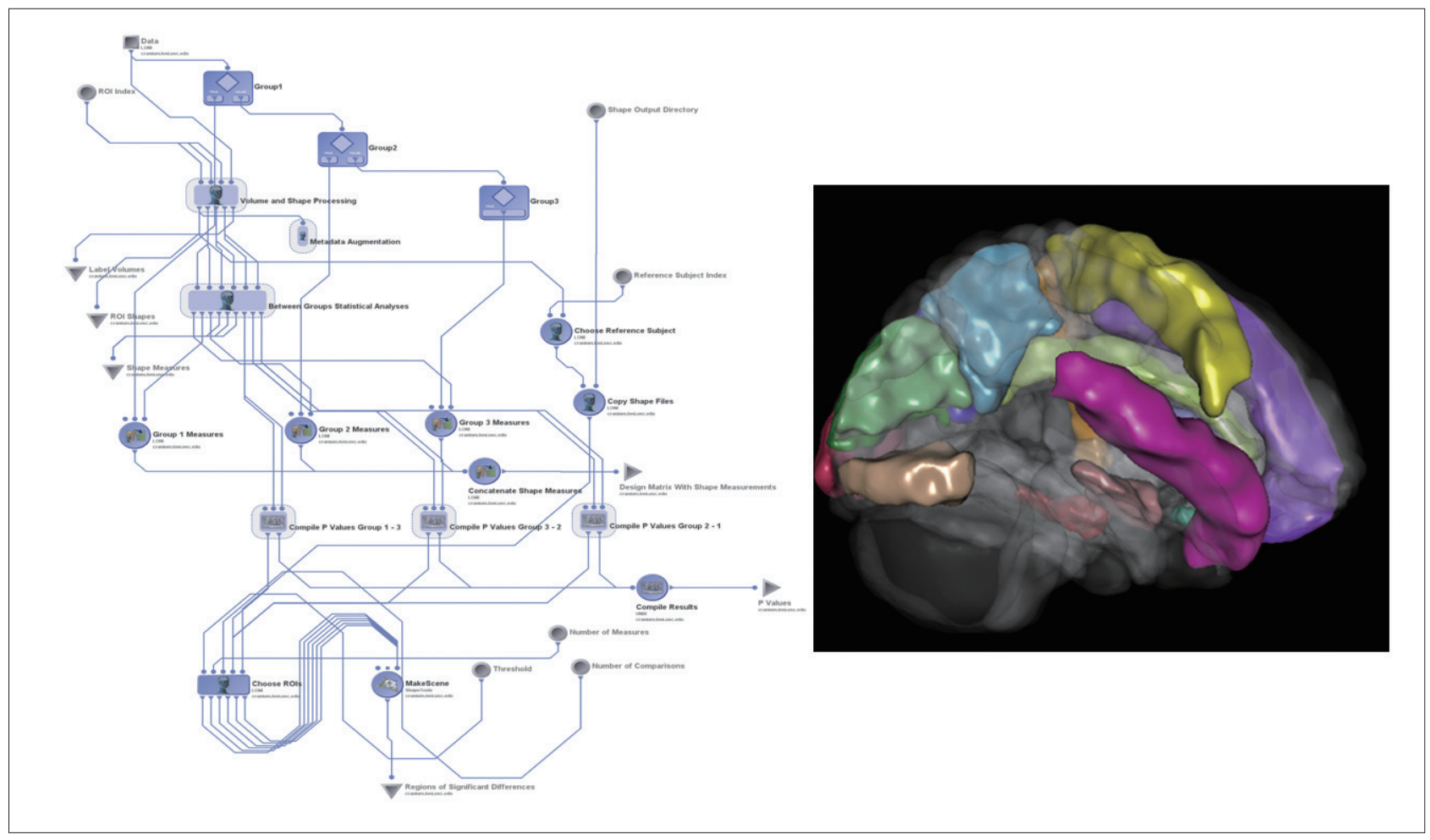

Figure 1. The global shape analysis (GSA) pipeline workflow and one example of a 3D scene output file indicating statistically significant $(p$-value $<0.05)$ volumetric differences between the early-onset Alzheimer's disease and early-onset mild cognitive impairment cohorts. These scene files are generated for each group comparison and each shape or volume metric.

tain a set of 15 neuroimaging biomarkers.

From the collection of 280 imaging markers (56 ROIs $x$ five shape measures), we chose the 15 most significant neuroimaging biomarkers that provided the highest discrimination between the EO-AD and EO-MCI groups. We selected 15 neuroimaging phenotypes using t-tests that compared the $\mathrm{EO}-\mathrm{AD}$ and $\mathrm{EO}-\mathrm{MCI}$ groups (with an a priori false-positive rate of 0.05). These biomarkers are described in Table 1. The 15 neuroimaging biomarkers were derived from the structural imaging data using the GSA workflow and are based on the automated ROI extractions generated by BrainParser. ${ }^{14,15}$ Figure 2 illustrates the LPBA40 atlas, an example of the 3D reconstruction of the BrainParser output for one subject, and the names of the 56 ROIs are shown in Table 2.

Then the 15 neuroimaging biomarkers were associated with the top 20 SNPs that were chosen with PLINK. The Pipeline workflow, as shown in Figure 3A and B, provided the most significant genotypic discriminants between the EO-AD and $\mathrm{EO}-\mathrm{MCI}$ subjects. Figure $3 \mathrm{~A}$ shows the quality control pipeline workflow, and Figure $3 \mathrm{~B}$ illustrated the genetic association study workflow.

\section{Genetic association analysis}

We used standard genome-wide association study (GWAS) techniques ${ }^{31-33}$ to extract 20 SNPs according to their $\mathrm{p}$-values that showed significant differences between the EO-AD and EO-MCI subjects. The results of the association between the 20 SNPs phenotypes and the 15 neuroimaging biomarkers are shown using circular connections ${ }^{34}$ and bubble ${ }^{35}$ charts.

\section{RESULTS}

\section{Demographic characteristics}

The demographic and clinical data of the subjects at the baseline are summarized in Table 3 (using chi-square and ttest analyses). EO subjects (aged between 55 and 65 years) were chosen from the 589 ADNI datasets. There was no statistically significant difference in age between the EO-AD and EO-MCI subjects.

\section{SNP phenotypes and neuroimaging biomarker selection}

The QC results are shown in Figure 4 and the 20 SNPs that were chosen according to their p-values are shown in Figure 5 and Table 1 . The QQ normal probability plot is shown in Figure 6. The 15 most significant neuroimaging biomarkers were selected from among the 56 ROIs and five different volume- and shape-based metrics, based on how well they discriminated between the two cohorts in Table 1. 
Table 1. Summary of the most significant genetics and imaging phenotypes - 15 derived-bioimaging markers and the 20 SNPs

\begin{tabular}{|c|c|c|c|c|c|c|}
\hline Neuroimaging phenotypes & p-value & Index & SNPs & Chromosome & p-value & Gene \\
\hline $\begin{array}{l}\text { L_cingulate_gyrus } \\
\text { (Average mean curvature) }\end{array}$ & 0.0335 & 1 & rs17029131 & 2 & $3.52 \mathrm{E}-06$ & \\
\hline L_gyrus_rectus (Surface area) & 0.01728 & 2 & rs1822144 & 2 & $2.28 \mathrm{E}-06$ & \\
\hline R_cuneus (Surface area) & 0.04203 & 3 & rs6446443 & 4 & $6.68 \mathrm{E}-05$ & $\begin{array}{l}\text { JAKMIP1 (janus kinase \& } \\
\text { microtubule interacting protein } 1 \text { ) }\end{array}$ \\
\hline R_superior_frontal_gyrus (Volume) & 0.03706 & 4 & rs12506164 & 4 & $1.75 \mathrm{E}-05$ & \\
\hline L_precentral_gyrus (Volume) & 0.04125 & 5 & rs7718456 & 5 & $3.36 \mathrm{E}-05$ & \\
\hline L_precuneus (Volume) & 0.0508 & 6 & rs9377090 & 6 & $3.36 \mathrm{E}-05$ & \\
\hline $\begin{array}{l}\text { L_middle_occipital_gyrus } \\
\text { (Volume) }\end{array}$ & 0.01805 & 7 & rs2776932 & 10 & $2.20 \mathrm{E}-05$ & NRP1 (neuropilin1) \\
\hline $\begin{array}{l}\text { R_superior_temporal_gyrus } \\
\text { (Volume) }\end{array}$ & 0.03353 & 8 & rs4933672 & 10 & $6.48 \mathrm{E}-05$ & \\
\hline L_hippocampus (Volume) & 0.00067 & 9 & rs11193270 & 10 & $3.52 \mathrm{E}-06$ & \\
\hline R_hippocampus (Volume) & 0.00539 & 10 & rs11193272 & 10 & $3.52 \mathrm{E}-06$ & \\
\hline R_precentral_gyrus (Shape index) & 0.03411 & 11 & rs11193274 & 10 & $3.52 \mathrm{E}-06$ & \\
\hline R_precuneus (Shape index) & 0.03186 & 12 & rs12218153 & 10 & $3.52 \mathrm{E}-06$ & \\
\hline L_cuneus (Shape index) & 0.04952 & 13 & rs1338956 & 10 & $2.20 \mathrm{E}-05$ & \\
\hline $\begin{array}{l}\text { R_inferior_occipital_gyrus } \\
\text { (Curviness) }\end{array}$ & 0.05037 & 14 & rs1338025 & 10 & $2.20 \mathrm{E}-05$ & \\
\hline \multirow[t]{6}{*}{ R_putamen (Curviness) } & 0.03504 & 15 & rs12101936 & 15 & $7.08 \mathrm{E}-06$ & \\
\hline & & 16 & rs16964473 & 19 & $3.53 \mathrm{E}-05$ & Intergenic \\
\hline & & 17 & rs12972537 & 19 & $6.14 \mathrm{E}-05$ & \\
\hline & & 18 & rs2212356 & 21 & $6.23 \mathrm{E}-05$ & \\
\hline & & 19 & rs2831165 & 21 & $6.68 \mathrm{E}-05$ & \\
\hline & & 20 & rs 1266320 & 23 & $4.46 \mathrm{E}-06$ & \\
\hline
\end{tabular}

SNP: single nucleotide polymorphism, CHR: chromosome

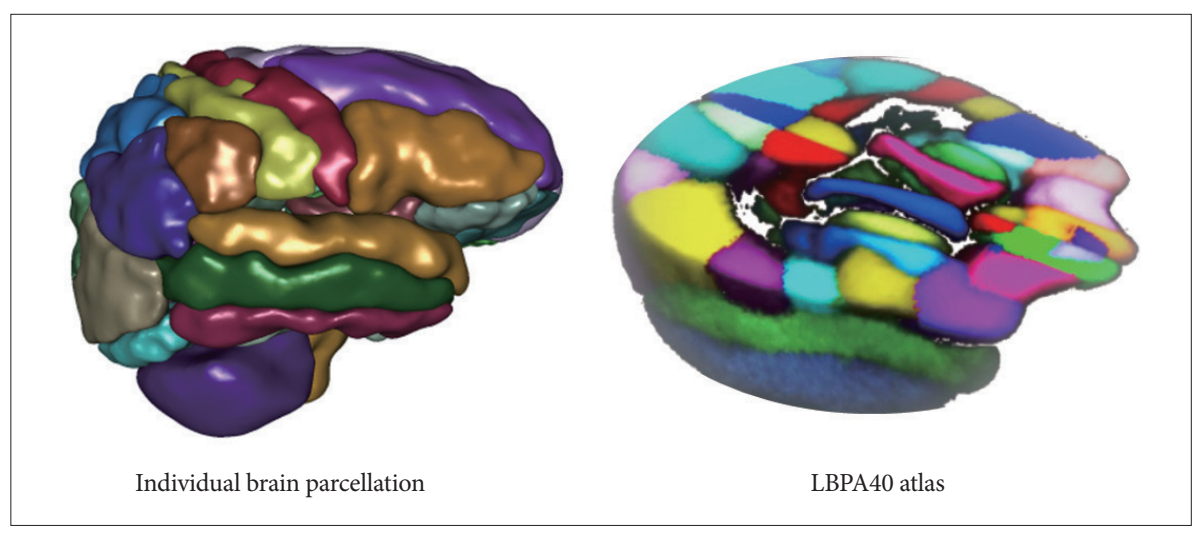

Figure 2. Individual brain parcellation and LONI Probabilistic Brain Atlas (LPBA40) atlas.

\section{Genetic association study}

The genetic association study between the 20 SNPs and the 15 neuroimaging phenotypes are shown in Figure 7. The Pipeline workflow used to compute these SNP-imaging biomarker associations is shown in Figure 3B.

In the association results of all 36 subjects, as shown in Figure $7 \mathrm{~A}$, there were several significant results $(\mathrm{p}<0.01)$ such as between rs7718456 (Chr 15) and L_rectus_gyrus (surface area) ( $\mathrm{p}=0.00964)$; rs7718456 (Chr 15) and R_superior_frontal_gyrus (volume) ( $\mathrm{p}=0.00867)$; rs7718456 (Chr 15) and L_ hippocampus (volume) ( $\mathrm{p}=0.00566) ;$ rs7718456 ( $\mathrm{Chr} 15)$ and R_hippocampus (volume) ( $\mathrm{p}=0.000618) ;$ rs4933672 (Chr 10) and R_precuneus (shape index) ( $\mathrm{p}=0.00476)$; and rs7718456 (Chr 5) and L_cuneus (shape index) $(\mathrm{p}=0.00125)$. 
Table 2. The names of the $56 \mathrm{RO}$ s

\begin{tabular}{|c|c|c|}
\hline Index & Volume_intensity & ROI_name \\
\hline 0 & 0 & Background \\
\hline 1 & 21 & L_superior_frontal_gyrus \\
\hline 2 & 24 & R_middle_frontal_gyrus \\
\hline 3 & 50 & R_precuneus \\
\hline 4 & 181 & Cerebellum \\
\hline 5 & 47 & L_angular_gyrus \\
\hline 6 & 122 & R_cingulate_gyrus \\
\hline 7 & 83 & L_middle_temporal_gyrus \\
\hline 8 & 90 & R_lingual_gyrus \\
\hline 9 & 81 & L_superior_temporal_gyrus \\
\hline 10 & 91 & L_fusiform_gyrus \\
\hline 11 & 44 & R_superior_parietal_gyrus \\
\hline 12 & 66 & R_inferior_occipital_gyrus \\
\hline 13 & 87 & L_parahippocampal_gyrus \\
\hline 14 & 162 & R_caudate \\
\hline 15 & 85 & L_inferior_temporal_gyrus \\
\hline 16 & 182 & Brainstem \\
\hline 17 & 43 & L_superior_parietal_gyrus \\
\hline 18 & 28 & R_precentral_gyrus \\
\hline 19 & 23 & L_middle_frontal_gyrus \\
\hline 20 & 89 & L_lingual_gyrus \\
\hline 21 & 41 & L_postcentral_gyrus \\
\hline 22 & 86 & R_inferior_temporal_gyrus \\
\hline 23 & 163 & L_putamen \\
\hline 24 & 26 & R_inferior_frontal_gyrus \\
\hline 25 & 102 & R_insular_cortex \\
\hline 26 & 25 & L_inferior_frontal_gyrus \\
\hline 27 & 46 & R_supramarginal_gyrus \\
\hline 28 & 34 & R_gyrus_rectus \\
\hline 29 & 65 & L_inferior_occipital_gyrus \\
\hline 30 & 164 & R_putamen \\
\hline 31 & 61 & L_superior_occipital_gyrus \\
\hline 32 & 30 & R_middle_orbitofrontal_gyrus \\
\hline 33 & 42 & R_postcentral_gyrus \\
\hline 34 & 27 & L_precentral_gyrus \\
\hline 35 & 32 & R_lateral_orbitofrontal_gyrus \\
\hline 36 & 121 & L_cingulate_gyrus \\
\hline 37 & 31 & L_lateral_orbitofrontal_gyrus \\
\hline 38 & 92 & R_fusiform_gyrus \\
\hline 39 & 45 & L_supramarginal_gyrus \\
\hline 40 & 88 & R_parahippocampal_gyrus \\
\hline 41 & 22 & R_superior_frontal_gyrus \\
\hline 42 & 29 & L_middle_orbitofrontal_gyrus \\
\hline 43 & 68 & R_cuneus \\
\hline
\end{tabular}

Table 2. The names of the 56 ROIs (continued)

\begin{tabular}{ccl}
\hline Index & Volume_intensity & \multicolumn{1}{c}{ ROI_name } \\
\hline 44 & 62 & R_superior_occipital_gyrus \\
45 & 33 & L_gyrus_rectus \\
46 & 48 & R_angular_gyrus \\
47 & 64 & R_middle_occipital_gyrus \\
48 & 84 & R_middle_temporal_gyrus \\
49 & 49 & L_precuneus \\
50 & 67 & L_cuneus \\
51 & 161 & L_caudate \\
52 & 165 & L_hippocampus \\
53 & 166 & R_hippocampus \\
54 & 82 & R_superior_temporal_gyrus \\
55 & 63 & L_middle_occipital_gyrus \\
56 & 101 & L_insular_cortex \\
\hline
\end{tabular}

ROI: region of interest

In the association results of the $27 \mathrm{MCI}$ subjects, as shown in Figure 7B, there were several significant results $(\mathrm{p}<0.001)$ such as between rs9377090 (Chr 6) and L_rectus_gyrus (surface area) ( $\mathrm{p}=0.000121) ; \mathrm{rs} 2212356$ (Chr 21) and R_cuneus (surface area) ( $\mathrm{p}=0.000131) ; \mathrm{rs} 17029131$ (Chr 2) and R_superior_frontal_gyrus (volume) $(\mathrm{p}<0.000950) ;$ rs6446443 (Chr $4)$ and R_superior_frontal_gyrus (volume) $(\mathrm{p}=0.000005269)$; rs 17029131 (Chr 2) and L_Precuneus (volume) ( $\mathrm{p}=$ 0.000000008832); rs9377090 (Chr 6) and L_Precuneus (volume) ( $\mathrm{p}=0.000371)$; rs7718456 (Chr 5) and R_superior_temporal_gyrus (volume) $(\mathrm{p}=0.000086) ;$ rs 11193270 (Chr 10) and R_superior_temporal_gyrus (volume) $(\mathrm{p}=0.000748)$; rs11193272 (Chr 10) and R_superior_temporal_gyrus (volume) ( $\mathrm{p}=0.000748)$; rs11193274 (Chr 10) and R_superior_ temporal_gyrus (volume) $(\mathrm{p}=0.000748) ;$ rs12218153 (Chr 10) and R_superior_temporal_gyrus (volume) $(\mathrm{p}=0.000748)$; rs1338956 (Chr 10) and R_superior_temporal_gyrus (volume) ( $\mathrm{p}=0.000748)$; rs1338025 (Chr 10) and R_superior_ temporal_gyrus (volume) ( $\mathrm{p}=0.000748) ; \mathrm{rs} 9377090$ (Chr 6) and R_Hippocampus (volume) ( $\mathrm{p}=0.000006)$; rs17029131 (Chr 2) and R_precentral_gyrus (shape index) $(\mathrm{p}=0.000290)$; rs7718456 (Chr 5) and L_Cuneus (shape index) $(\mathrm{p}=0.000024)$; and rs2831165 and R_Putamen (curvedness) ( $\mathrm{p}=0.000232$ ).

In the association results of the nine $\mathrm{AD}$ subjects, as shown in Figure $7 \mathrm{C}$, there were several significant results $(\mathrm{p}<0.009)$ such as between rs16964473 (Chr 19) and L_rectus gyrus (surface area) ( $\mathrm{p}=0.00235)$; rs12972537 (Chr 19) and L_rectus_gyrus (surface area) ( $\mathrm{p}=0.00235)$; rs7718456 (Chr 5) and R_hippocampus (volume) ( $\mathrm{p}=0.00272) ;$ rs1266320 (Chr 23) and R_hippocampus (volume) $(\mathrm{p}=0.00275) ; \mathrm{rs} 1266320$ (Chr 23) and L_cuneus (shape index) ( $\mathrm{p}=0.00537) ; \mathrm{rs} 12101936$ (Chr 15) and R_inf_occipital_gyrus (curvedness) ( $\mathrm{p}=0.00138$ ); 
Table 3. Demographic information

\begin{tabular}{clccc}
\hline Cohort & Demographics & AD & MCI & p-value \\
\hline EO (36) & No. of subjects & 9 & 27 & - \\
& Age & $60.4 / 3.34$ & $61.2 / 2.87$ & 0.0810 \\
& Gender (M/F) & $4 / 5$ & $15 / 12$ & 0.5630 \\
& Education & $16.142 \pm 2.304$ & $16.226 \pm 2.764$ & 0.8834 \\
& MMSE & $21.571 \pm 3.795$ & $26.745 \pm 2.342$ & $<0.0001$ \\
& Handedness (R/L) & $5 / 4$ & $24 / 3$ & 0.0286 \\
& ApoE (ع4) (+/-) & $5 / 4$ & $14 / 13$ & 0.8471 \\
\hline
\end{tabular}

EO: early-onset, AD: Alzheimer's disease, MCI: mild cognitive impairment, MMSE: Mini-Mental State Examination


Figure 5. Manhattan plot for all the single nucleotide polymorphism.

Figure 3. Plink workflows. A: The pipeline workflow for quality control. B: Genetic association study.

Figure 4. Quality control process. 
and rs6446443 (Chr 4) and R_putamen (curvedness) ( $\mathrm{p}=$ $0.00821)$.

In addition, we used dynamic circular connection graphs to illustrate the relationship between the significant SNPs and

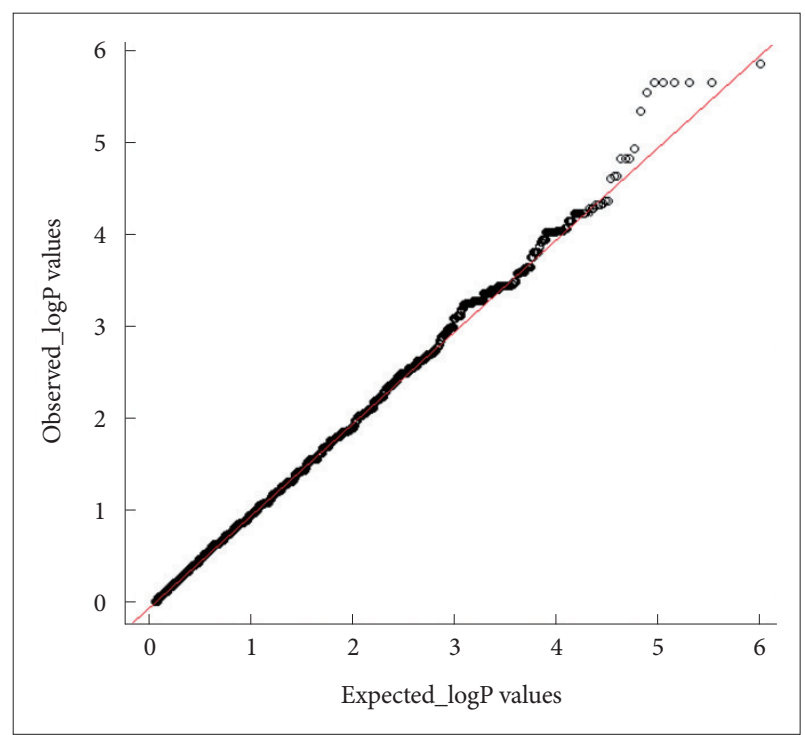

the neuroimaging biomarkers, as shown in Figure 8. The left and right semicircles of the connectogram graph contain the 15_ROI imaging markers and the 20_SNPs genotypes, respectively. The width and brightness of the ribbon connecting each SNP-ROI pair presents the strength of the phenotypegenotype association.

\section{DISCUSSION}

\section{Demographic characteristics}

The demographic and clinical data of the subjects at the baseline are summarized in Table 1 (using chi square and ttest statistics). We chose EO subjects who were between 55 and 65 years old from the entire 589 ADNI data set. There were significant differences in gender, MMSE score, and APOE status between total AD and total MCI subjects. On the other hand, between the EO-AD and EO-MCI subjects, there was no significant difference in age, gender, years of education, handedness, and APOE status, but the MMSE scores significantly differed, as we expected.

Figure 6. QQ normal probability plot.

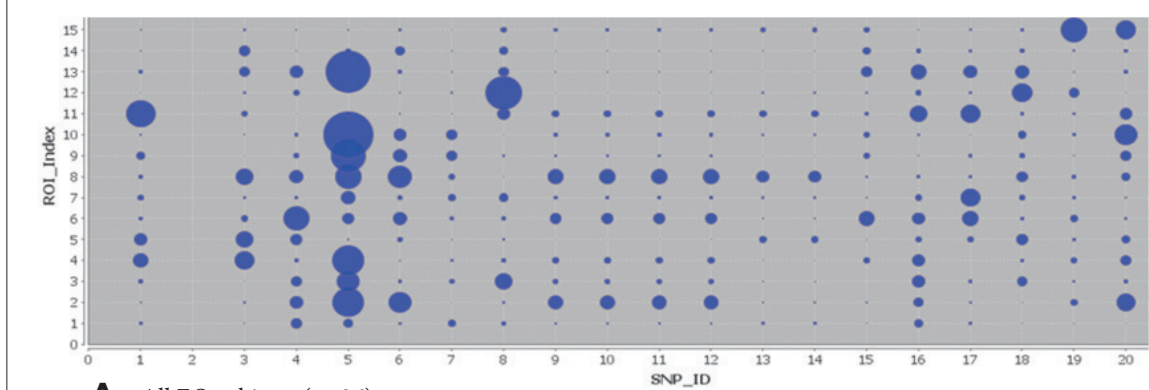

A All EO subjects $(n=36)$

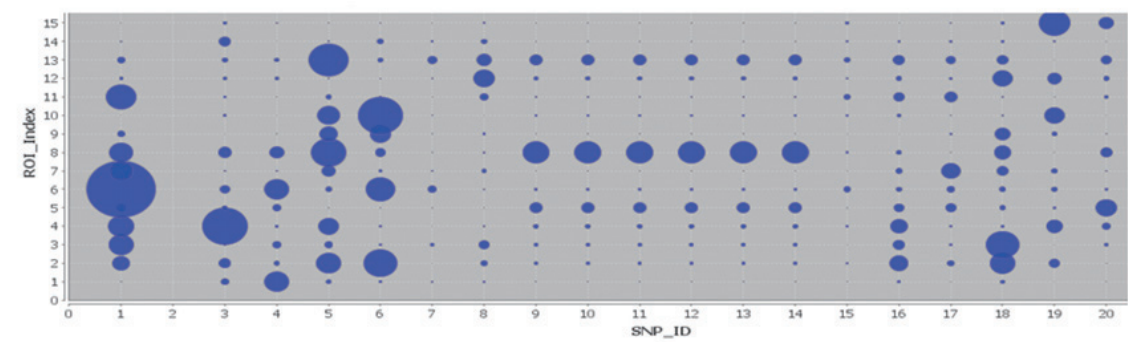

B MCI subjects $(\mathrm{n}=27)$

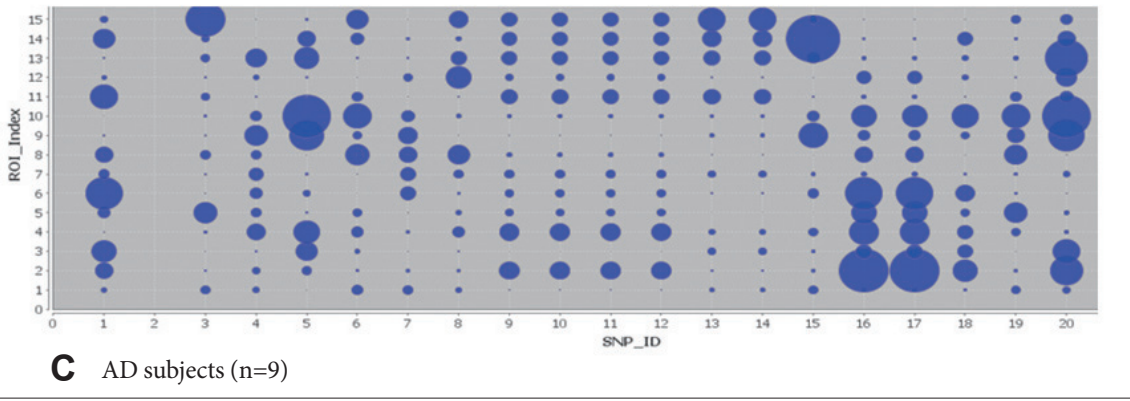

Figure 7. A: There were several significant results $(p<0.001)$ such as between rs17029131 and L_Precuneus (volume) $(p=0.000000008832) ; r 6446443$ and R_superior_frontal_gyrus (volume) $(p=0.000005269) ;$ rs9377090 and $R$ Hippocampus (volume) $(p=0.000006)$; rs7718456 and L Cuneus (shape index) $(p=0.000024)$; and rs7718456 and $\mathrm{R}$ superior temporal gyrus (volume) $(p=0.000086)$. B: There were several significant results $(p<0.001)$ such as between rs17029131 and L_Precuneus (volume) $(p=0.000000008832)$; rs6446443 and R_superior_frontal_gyrus (volume) $(\bar{p}=0.000005269)$; rs9377090 and R_Hippocampus (volume) $(p=0.000006)$; rs7718456 and $L_{-}$ Cuneus (shape index) $(p=0.000024)$; and rs7718456 and R_superior_temporal_gyrus (volume) $(\bar{p}=0.000086)$. C: There were several significant results $(p<0.009)$ such as between rs12101936 and R_inf_occipital_gyrus (curvedness) $(p=0.00138) ; r s 16964473$ and $L$ rectus gyrus (surface area) $(p=0.00235)$; rs12972537 and L_rectus_gyrus (surface area) ( $p=0.00235)$; rs7718456 and R_hippocampus (volume) $(p=0.00272)$; and rs1266320 and R_hippocampus (volume) $(p=0.00275)$. 


\section{SNP selection}

A total of $36 \mathrm{EO}$ individuals enrolled in this study had genotype data available for analysis without exclusion. The flow sheet for the workflow is shown in Figure 3. We obtained the 367,899 SNPs from 620,901 SNPs via the LONI pipeline QC workflow (Figure 4A is for the QC workflow). These SNPs were selected by computing their association with the diagnosis of $\mathrm{AD}$ or $\mathrm{MCI}$ as a dependent variable using PLINK. We generated a Manhattan plot to see which SNPs were associated with the diagnosis of MCI or AD. From this plot, we were able to derive the 20 most significant SNPs, as shown in Table 2. The QQ normal probability plot (Figure 6) shows us that the EO-AD and EO-MCI groups significantly differed in their genetic findings of cognitive impairment. Two SNPs were associated with genes such as rs6446443 for the JAKMIP1 (janus kinase and microtubule interacting protein 1) gene and rs2776932 for the NRP1 (neuropilin-1) gene. The JAKMIP1 gene is associated with microtubules and may play a role in the microtubule-dependent transport of the GABA$B$ receptor and in JAK1 signaling and regulation of microtubules cytoskeleton rearrangements. ${ }^{36}$ The NRP1 (neutrophilin-1) gene plays versatile roles in angiogenesis, axon guidance, cell survival, migration, and invasion. ${ }^{37}$ These two genes were involved in the development of $\mathrm{MCI}$ to $\mathrm{AD}$ in a total of $36 \mathrm{EO}$

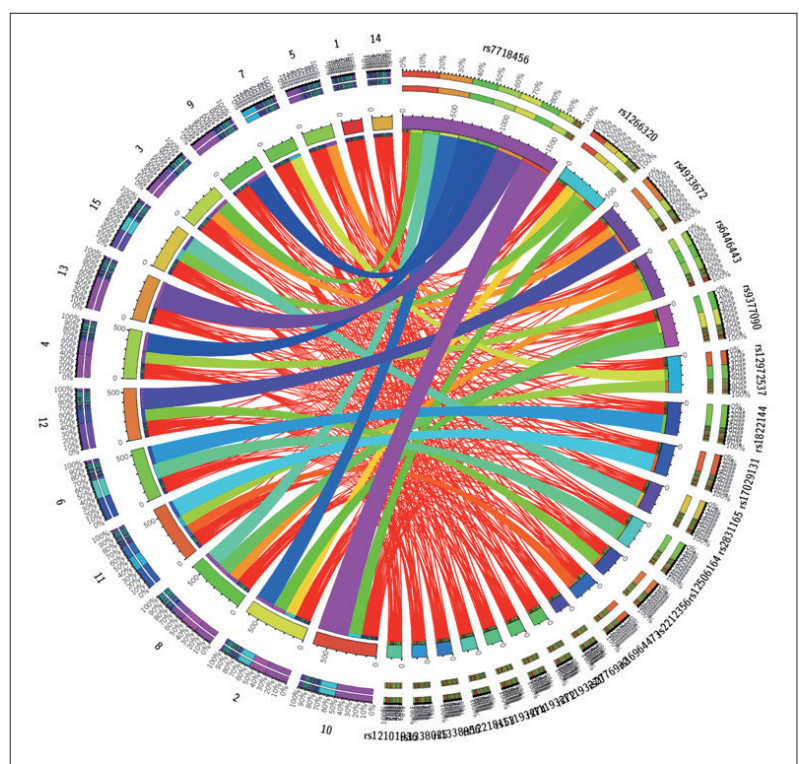

Figure 8. Circular representation of the significant SNP-Neuroimaging interactions. The left and right parts of the graph contain the 15-ROI imaging markers and the 20-SNP genotypes, respectively. The strength of the connection between each SNP-ROI pair is presented as a ribbon, whose size, color and location are proportional to $-\log (p)$. Clearly, there are a lot of spurious effects (skinny red lines on background) and several significantly strong associations (thicker purple ribbons on foreground), e.g., purple association between SNP_5 (rs7718456) and ROI_10 (L_hippocampus, Volume). SNP: single nucleotide polymorphism, $\bar{R}$ OI: region of interest. individuals in this EO cognitive impairment study.

\section{Shape measures}

Table 4 defines the five intrinsic geometric cortical measures used in this study, and the formulas used to compute them. The principal curvatures ( $\kappa_{1}$ and $\kappa_{2}$ ) were computed using triangulated surface models that represented the boundaries of different brain areas. ${ }^{38} \mathrm{I}_{\mathrm{D}}(\mathrm{x}, \mathrm{y}, \mathrm{z})$ represents the indicator function of the region of interest (D), ${ }^{39}$ and $\mathrm{S}_{\Omega}: \mathrm{r}=\mathrm{r}(\mathrm{u}$, $v)$ and (u,v) $\in \Omega$ is the parametric surface representation of the region boundary. ${ }^{40}$ In the local shape analysis (LSA) protocol, the structural attributes and cortical measures were calculated per vertex in specific shape regions that were first co-registered across the subjects to establish homologous anatomical features before statistically analyzing them against various demographic, clinical or phenotypic subject data. ${ }^{28}$

\section{Global shape analysis}

The left and right hippocampal volumes were the most significant neuroimaging biomarkers, as we expected. The available data seem to suggest that evaluating hippocampal atrophy may be helpful in EO-AD, ${ }^{41}$ but it is difficult to say that hippocampal volumes have closer relationships in EO-AD than in LO-AD because we did not directly compare the two groups. It has been reported that $\mathrm{EO}-\mathrm{AD}$ and $\mathrm{LO}-\mathrm{AD}$ can differ in their typical topographic patterns of brain atrophy, which suggests that the neocortical functions can be more affected in EO-AD. ${ }^{41,42}$ The left precuneus volume was significantly reduced in the EO group, which supports the findings of some previously mentioned studies, which may explain why precuneal atrophy is more prominent in patients with EO than in patients with $\mathrm{LO}^{43,44}$ The precuneus is located beside the temporo-parietal junction, where greater neocortical atrophy is reported in EO-AD than in LO-AD.$^{41} \mathrm{In}$ this study, the 'shape index' measure of the right precuneus was also significant, which indicates the possibility of various shape-metric changes in the precuneal region. The 'average mean curva-

Table 4. Intrinsic geometric cortical features and their definitions

\begin{tabular}{lc}
\hline Geometric measure & Mathematical formulas \\
\hline Volume & $\iiint_{R^{3}} I_{D}(x, y, z) d x d y d z$ \\
Surface area & $\iint_{\Omega}\left|\vec{r}_{u} \times \vec{r}_{v}\right| d u d v$ \\
Mean curvature & $\frac{1}{2}\left(\kappa_{1}+\kappa_{2}\right)$ \\
Shape index & $\frac{2}{\pi} \arctan \left(\frac{\kappa_{2}+\kappa_{1}}{\kappa_{2}-\kappa_{1}}\right)$ \\
Curvedness & $\sqrt{\frac{\kappa_{1}^{2}+\kappa_{2}^{2}}{2}}$ \\
\hline
\end{tabular}


ture' metric indicated that the left cingulated gyrus and the left superior temporal gyrus significantly differed between EO and $\mathrm{LO}$, which suggests that $\mathrm{EO}-\mathrm{AD}$ differences may be preferentially related to atrophy of the posterior cingulated cortex and the temporal lobe. ${ }^{45}$

\section{Genetic Association Study (GWAS using selected SNPs and shape measures)}

We can see all the results in the bubble charts (Figure 7) and the circular representation (Figure 8). The bubble size increased according to the increase in the significance of the association between the neuroimaging phenotypes and the SNPS. The stripes in the circus could be seen better from the foreground as the significance increased. The results for all the $27 \mathrm{MCI}$ subjects and nine $\mathrm{AD}$ subjects show that several associations between the neuroimaging genetics and the SNPs were statistically significant, although they could not be survived by FDR correction. The left and right hippocampal volumes, and the left precuneal volume and the right precuneal shape index, were as significantly associated as the neuroimaging phenotypes in this analysis. In this study, the JAKMIP1 gene (rs6446443) and the NRP1 gene (rs2776932) were associated with the neuroimaging and genetic phenotypes. For all the 36 subjects, SNP rs7718456 at chromosome 5 was most significantly associated with both hippocampi volumes. For the 27 EO-MCI subjects, SNP rs17029131 at chromosome 2 was most significantly related to the left precuneus volume. For the nine EO-AD subjects, SNP rs1822144 at chromosome 2 was significantly associated with the left precuneus volume. Similar to these, both hippocampal volumes and the left precuneus volume were significantly associated with specific SNPs. Thus, we could conclude that the hypotheses we mentioned in the introduction correspond with these results. We expect to validate these results using a large population of EO cognitive impairment patients and to compare them with the results for LO cognitive impairment patients in terms of neuroimaging genetics.

\section{Limitations}

In this EO study, the sample size was rather small due to significant data stratification and lack of available data. This contributed to the relative weakness of the statistical results. The small sample size also kept us from statistically analyzing individual APOE 2, 3, and 4 alleles. Since the ADNI did not include genetic information for the normal controls, we had no way of comparing the $\mathrm{AD}$ and $\mathrm{MCI}$ subjects against agematched healthy individuals. Even with the small number of EO subjects, though, we were able to make several neuroimaging genetics observations regarding the $\mathrm{EO}-\mathrm{AD}$ and $\mathrm{EO}-$ MCI cohorts. In this study, we developed a protocol for per- forming neuroimaging genetics analysis using the LONI Pipeline environment. The methodology presented here can be used as a basis for future large-scale neuroimaging genetics studies.

\section{Acknowledgments}

Data used in preparation of this article were obtained from the Alzheimer's Disease Neuroimaging Initiative (ADNI) database (http://ADNI.loni. usc.edu). As such, the investigators within the ADNI contributed to the design and implementation of ADNI and/or provided data but did not participate in analysis or writing of this report. A complete listing of ADNI investigators can be found at: http://ADNI.loni.usc.edu/study-design/ongoing-investigations/.

The study design, data analyses and writing of this manuscript were supported in part by NIA P 50 AG16570, NIBIB EB01651, NLM LM05639, NCRR RR019771, NIMH R01 MH071940, NIBIB 9P41EB015922-15, NCRR 2-P41-RR-013642-15, NCRR U54 RR021813, U24-RR025736, U24RR021992, P20 NR015331, U54 EB020406, P50 NS091856, P30 DK089503 as well as NSF grants 0716055 and 1023115 .

This research was partially supported by Basic Science Research Program through the National Research Foundation of Korea(NRF) funded by the Ministry of Education(NRF-2012R1A1A4A01013120).

Data collection and sharing for this project was funded by the Alzheimer's Disease Neuroimaging Initiative (ADNI) (National Institutes of Health Grant U01 AG024904). ADNI is funded by the National Institute on Aging, the National Institute of Biomedical Imaging and Bioengineering, and through generous contributions from the following: Abbott; Alzheimer's Association; Alzheimer's Drug Discovery Foundation; Amorfix Life Sciences Ltd.; AstraZeneca; Bayer HealthCare; BioClinica, Inc.; Biogen Idec Inc.; Bristol-Myers Squibb Company; Eisai Inc.; Elan Pharmaceuticals Inc.; Eli Lilly and Company; F. Hoffmann-La Roche Ltd and its affiliated company Genentech, Inc.; GE Healthcare; Innogenetics, N.V.; IXICO Ltd.; Janssen Alzheimer Immunotherapy Research \& Development, LLC.; Johnson \& Johnson Pharmaceutical Research \& Development LLC.; Medpace, Inc.; Merck \& Co., Inc.; Meso Scale Diagnostics, LLC.; Novartis Pharmaceuticals Corporation; Pfizer Inc.; Servier; Synarc Inc.; and Takeda Pharmaceutical Company. The Canadian Institutes of Health Research is providing funds to support ADNI clinical sites in Canada. Private sector contributions are facilitated by the Foundation for the National Institutes of Health (www.fnih. org). The grantee organization is the Northern California Institute for Research and Education, and the study is Rev March 26, 2012 coordinated by the Alzheimer's Disease Cooperative Study at the University of California, San Diego. ADNI data are disseminated by the Laboratory for Neuro Imaging at the University of California, Los Angeles. This research was also supported by NIH grants P30 AG010129 and K01 AG030514.

\section{REFERENCES}

1. Gatz M, Reynolds CA, Fratiglioni L, Johansson B, Mortimer JA, Berg S, et al. Role of genes and environments for explaining Alzheimer disease. Arch Gen Psychiatry 2006;63:168-174.

2. Ertekin-Taner N. Genetics of Alzheimer's Disease: a centennial review. Neurol Clin 2007;25:611-667.

3. Goate A, Chartier-Harlin MC, Mullan M, Brown J, Crawford F, Fidani $\mathrm{L}$, et al. Segregation of a missense mutation in the amyloid precursor protein gene with familial Alzheimer's disease. Nature 1991;349:704706.

4. Sherrington R, Rogaev EI, Liang Y, Rogaeva EA, Levesque G, Ikeda M, et al. Cloning of a gene bearing missense mutations in early-onset familial Alzheimer's disease. Nature 1995;375:754-760.

5. Levy-Lahad E, Wasco W, Poorkaj P, Romano DM, Oshima J, Pettingell $\mathrm{WH}$, et al. Candidate gene for the chromosome 1 familial Alzheimer's disease locus. Science 1995;269:973-977.

6. Rogaev EI, Sherrington R, Rogaeva EA, Levesque G, Ikeda M, Liang Y, 
et al. Familial Alzheimer's disease in kindreds with missense mutations in a gene on chromosome 1 related to the Alzheimer's disease type 3 gene. Nature 1995;376:775-778.

7. Campion D, Dumanchin C, Hannequin D, Dubois B, Belliard S, Puel $\mathrm{M}$, et al. Early-onset autosomal dominant Alzheimer disease: prevalence, genetic heterogeneity, and mutation spectrum. Am J Hum Genet 1999;65:664-670.

8. Toga AW. Neuroimage databases: the good, the bad and the ugly. Nature reviews. Nat Rev Neurosci 2002;3:302-309.

9. Stein JL, Hua X, Lee S, Ho AJ, Leow AD, Toga AW, et al. Voxelwise genome-wide association study (vGWAS). Neuroimage 2010;53:11601174.

10. Potkin SG, Guffanti G, Lakatos A, Turner JA, Kruggel F, Fallon JH, et al. Hippocampal atrophy as a quantitative trait in a genome-wide association study identifying novel susceptibility genes for Alzheimer's disease. PLoS ONE 2009;4:e6501.

11. Glahn DC, Paus T, Thompson PM. Imaging genomics: mapping the influence of genetics on brain structure and function. Hum Brain Mapp 2007;28:461-463.

12. Cannon TD, Thompson PM, van Erp TG, Huttunen M, Lonnqvist J, Kaprio J, et al. Mapping heritability and molecular genetic associations with cortical features using probabilistic brain atlases: methods and applications to schizophrenia. Neuroinformatics 2006;4:5-19.

13. Potkin SG, Turner JA, Guffanti G, Lakatos A, Torri F, Keator DB, et al. Genome-wide strategies for discovering genetic influences on cognition and cognitive disorders: methodological considerations. Cogn Neuropsychiatry 2009;14:391-418.

14. Shattuck DW, Mirza M, Adisetiyo V, Hojatkashani C, Salamon G, Narr $\mathrm{KL}$, et al. Construction of a 3D probabilistic atlas of human cortical structures. Neuroimage 2008;39:1064-1080.

15. Lai DM, Li H, Lee CC, Tzeng YS, Hsieh YH, Hsu WM, et al. Angiopoietin-like protein 1 decreases blood brain barrier damage and edema following focal cerebral ischemia in mice. Neurochem Int 2008;52:470477.

16. Dinov I, Lozev K, Petrosyan P, Liu Z, Eggert P, Pierce J, et al. Neuroimaging study designs, computational analyses and data provenance using the LONI pipeline. PLoS ONE 2010;5:e13070.

17. Dinov ID, Van Horn JD, Lozev KM, Magsipoc R, Petrosyan P, Liu Z, et al. Efficient, distributed and interactive neuroimaging data analysis using the LONI pipeline. Front Neuroinform 2009;3:22.

18. Shen L, Kim S, Risacher SL, Nho K, Swaminathan S, West JD, et al. Whole genome association study of brain-wide imaging phenotypes for identifying quantitative trait loci in $\mathrm{MCI}$ and $\mathrm{AD}$ : a study of the ADNI cohort. Neuroimage 2010;53:1051-1063.

19. Biffi A, Anderson CD, Desikan RS, Sabuncu M, Cortellini L, Schmansky N, et al. Genetic variation and neuroimaging measures in Alzheimer disease. Arch Neurol 2010;67:677-685.

20. Petersen RC, Aisen PS, Beckett LA, Donohue MC, Gamst AC, Harvey DJ, et al. Alzheimer's Disease Neuroimaging Initiative (ADNI): clinical characterization. Neurology 2010;74:201-209.

21. Morris JC. The Clinical Dementia Rating (CDR): current version and scoring rules. Neurology 1993;43:2412-2414.

22. Jack CR Jr, Bernstein MA, Fox NC, Thompson P, Alexander G, Harvey $\mathrm{D}$, et al. The Alzheimer's Disease Neuroimaging Initiative (ADNI): MRI methods. J Magn Reson Imaging 2008;27:685-691.

23. Dale AM, Fischl B, Sereno MI. Cortical surface-based analysis. I. Segmentation and surface reconstruction. Neuroimage 1999;9:179-194.

24. Fischl B, Sereno MI, Dale AM. Cortical surface-based analysis. II: Inflation, flattening, and a surface-based coordinate system. Neuroimage 1999;9:195-207.

25. Desikan RS, Cabral HJ, Fischl B, Guttmann CR, Blacker D, Hyman BT, et al. Temporoparietal MR imaging measures of atrophy in subjects with mild cognitive impairment that predict subsequent diagnosis of Alzheimer disease. AJNR Am J Neuroradiol 2009;30:532-538.

26. Fischl B, Salat DH, Busa E, Albert M, Dieterich M, Haselgrove C, et al. Whole brain segmentation: automated labeling of neuroanatomical structures in the human brain. Neuron 2002;33:341-355.

27. Fischl B, Dale AM. Measuring the thickness of the human cerebral cortex from magnetic resonance images. Proc Natl Acad Sci U S A 2000;97:11050-11055.

28. Dinov ID, Torri F, Macciardi F, Petrosyan P, Liu Z, Zamanyan A, et al. Applications of the pipeline environment for visual informatics and genomics computations. BMC Bioinformatics 2011;12:304.

29. Smith SM. Fast robust automated brain extraction. Hum Brain Mapp 2002;17:143-155.

30. Pieper S, Lorensen B, Schroeder W, Kikinis R. The NA-MIC Kit: ITK, VTK, pipelines, grids and 3D slicer as an open platform for the medical image computing community. Proc IEEE Int Symp Biomed Imaging 2006;698-701.

31. Hunter DJ, Kraft P, Jacobs KB, Cox DG, Yeager M, Hankinson SE, et al. A genome-wide association study identifies alleles in FGFR2 associated with risk of sporadic postmenopausal breast cancer. Nat Genet 2007; 39:870-874

32. Hibar DP, Stein JL, Kohannim O, Jahanshad N, Saykin AJ, Shen L, et al. Voxelwise gene-wide association study (vGeneWAS): multivariate gene-based association testing in 731 elderly subjects. Neuroimage 2011;56:1875-1891.

33. Stein JL, Hua X, Lee S, Ho AJ, Leow AD, Toga AW, et al. Voxelwise genome-wide association study (vGWAS). Neuroimage 2010;53:11601174.

34. Krzywinski MI, Schein JE, Birol I, Connors J, Gascoyne R, Horsman D, et al. Circos: an information aesthetic for comparative genomics. Genome Res 2009;19:1639-1645.

35. Al-Aziz J, Christou N, Dinov ID. SOCR Motion Charts: an efficient, open-source, interactive and dynamic applet for visualizing longitudinal multivariate data. J Stat Educ 2010;18:1-29.

36. Costa V, Conte I, Ziviello C, Casamassimi A, Alfano G, Banfi S, et al. Identification and expression analysis of novel Jakmip1 transcripts. Gene 2007;402:1-8.

37. Soker S, Takashima S, Miao HQ, Neufeld G, Klagsbrun M. Neuropilin-1 is expressed by endothelial and tumor cells as an isoform-specific receptor for vascular endothelial growth factor. Cell 1998;92:735-745.

38. Terzopoulos D. The computation of visible-surface representations. IEEE Trans Pattern Anal Mach Intell 1988:417-438.

39. Larson R, Edwards BH. Calculus. CA: Brooks/Cole Pub Co; 2009.

40. Santaló LA. Integral Geometry and Geometric Probability. Cambridge: Cambridge Univ Press; 2004.

41. Padovani A, Gilberti N, Borroni B. The usefulness of biological and neuroimaging markers for the diagnosis of early-onset Alzheimer's disease. Int J Alzheimers Dis 2011;2011:296-374.

42. Frisoni GB, Pievani M, Testa C, Sabattoli F, Bresciani L, Bonetti M, et al. The topography of grey matter involvement in early and late onset Alzheimer's disease. Brain 2007;130:720-730.

43. Karas G, Scheltens P, Rombouts S, van Schijndel R, Klein M, Jones B, et al. Precuneus atrophy in early-onset Alzheimer's disease: a morphometric structural MRI study. Neuroradiology 2007;49:967-976.

44. Moller C, Vrenken H, Jiskoot L, Versteeg A, Barkhof F, Scheltens P, et al. Different patterns of gray matter atrophy in early- and late-onset Alzheimer's disease. Neurobiol Aging 2013;34:2014-2022.

45. Shiino A, Watanabe T, Kitagawa T, Kotani E, Takahashi J, Morikawa S, et al. Different atrophic patterns in early- and late-onset Alzheimer's disease and evaluation of clinical utility of a method of regional z-score analysis using voxel-based morphometry. Dement Geriatr Cogn Disord 2008;26:175-186. 